\title{
Ride responses of macpherson suspension systems
}

\author{
Cheng-Chi $\mathrm{Yu}^{1}$ and Chih-Chi $\mathrm{Chiu}^{2, *}$ \\ ${ }^{1}$ Department of Vehicle Engineering, National Taipei University of Technology, Taiwan \\ ${ }^{2}$ Department of Vehicle Engineering, National Taipei University of Technology, Taiwan
}

\begin{abstract}
The main purpose of this study is to obtain more correct vehicle ride responses by using a nonlinear ride model considering the effect of Macpherson suspension geometry. Traditional ride model applied to analysis and controller design uses a two degree of freedom linear model, which includes sprung mass and unsprung mass and a spring and a damper vertically connect them. In fact, suspension components do not vertically position above the tire. The motions of body and tire are not going straight up and down. Therefore, the analysis results obtained by the simple model are often different from the experimental values of the actual vehicle. Because of the difference between simple model and actual vehicle, the control strategy almost cannot apply to actual vehicle. In order to understand the effect of suspension geometry on the vehicle ride responses and design a more practical control strategy, a nonlinear model including the geometric parameters of the suspension is constructed in this study. To estimate the initial equilibrium position of the suspension assembly under load, the static equilibrium analysis and mechanism motion analysis are synchronous implemented at the same time. The nonlinear model describes not only the relative position and velocity but also the force transmission between body and tire. Furthermore, by linearize this nonlinear model the development of control strategy for subsequent (semi) active suspension system could be expected.
\end{abstract}

\section{Introduction}

Currently, traditional suspension mathematical models are prevalent in analyzing and controlling the ride quality of vehicles [1-4], as shown in Fig. 1 [5]. However, suspension components such as springs, shock absorbers, and hydraulic cylinders are not installed directly above the vehicle tires or positioned perpendicular to the horizontal plane of the vehicle, nor do the vehicle body and tires exhibit a straight up-anddown motion (Fig. 2). Therefore, a simple straight upand-down suspension model cannot produce analysis results concurring with those of experiments with real vehicles. Consequently, control strategies formulated using such a model cannot attain favorable effects.

Several studies have considered adopting the wheel center rate, which is derived from the geometric effect of the suspension, to replace the spring rate in a simplified model [6-7], thereby reducing the difference between the simplified model and real situations. However, few studies have mentioned the equivalent damping coefficients or equivalent driving force of shock absorbers, which are related to the control strategies of active and semi-active suspension systems. To accurately determine the effect of suspension components (e.g., springs, shock absorbers, and hydraulic cylinders) on vehicle dynamic behavior and design practicable control strategies, this study constructed a nonlinear model incorporating suspension- related geometric parameters. Analyses on the static balance and mechanism kinematics were also performed to estimate the initial balanced positions of suspension components subjected to various loads. This enabled comprehensively describing how the relative positions (spring rate), velocity (damping force), and force transmission (active suspension) of suspension components affect the motion of the vehicle body (ride quality) and tires (grip). Finally, the nonlinear model was linearized to design the control scheme of semi-active suspension systems.

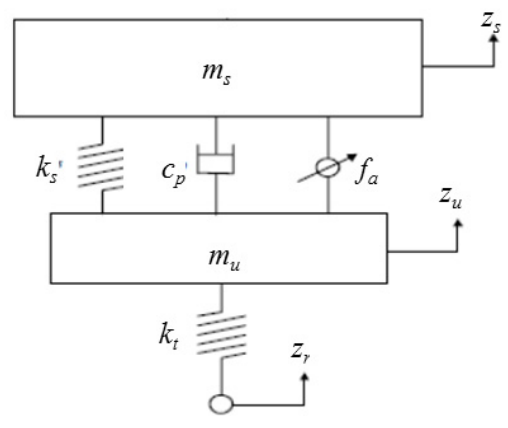

Fig. 1. Traditional suspension mathematical model [5].

\footnotetext{
Corresponding author: z175657@gmail.com
} 


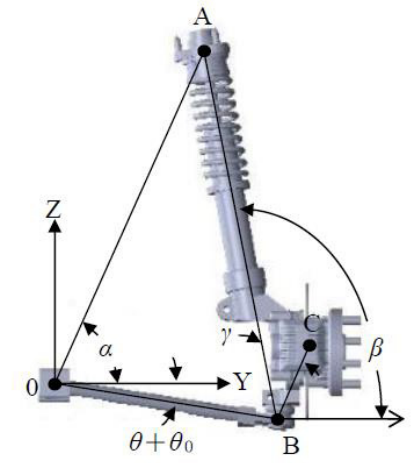

Fig. 2. Relative positions of suspension components in practice.

\section{Ride quality models}

\subsection{The $1 / 4$ vehicle suspension models}

Four 1/4 vehicle suspension models were constructed, namely, straight up-and-down (hereafter referred to as traditional), nonlinear, nonlinear linearized, and ADAMS 1/4 vehicle suspension models. In addition, changes in road surface conditions were used as input to compare the differences between the models in terms of various ride quality indicators, namely, vehicle body vertical acceleration, spring deformation, and tire deformation.

\subsubsection{Traditional suspension model}

Fig. 1 presents the schematic of a traditional suspension model, whose dynamic equation is expressed as Eq. (1). $m_{s}$ and $m_{u}$ denote the mass of the vehicle body (sprung mass) and wheel (unsprung mass), respectively. $k_{s}$ and $k_{t}$ represent the stiffness of the spring and tire, respectively. $c_{p}$ is the damping coefficient of the shock absorber. $z_{r}$ signifies the road surface deflection. $z_{s}$ and $z_{u}$ denote the vertical displacements of the vehicle body and tire, respectively.

$$
\begin{gathered}
m_{s} \ddot{z}_{s}=-k_{s}\left(z_{s}-z_{u}\right)-c_{p}\left(\dot{z}_{s}-\dot{z}_{u}\right) \\
m_{u} \ddot{z}_{u}=k_{s}\left(z_{s}-z_{u}\right)+c_{p}\left(\dot{z}_{s}-\dot{z}_{u}\right)-k_{t}\left(z_{u}-z_{r}\right)
\end{gathered}
$$

Rearranging Eq. (1) yields Eq. (2), where $x=\left[\begin{array}{lll}x_{1} & x_{2} & x_{3}\end{array}\right.$ $\left.x_{4}\right]^{T}$ represents the state vector, $x_{1}=z_{s}-z_{u}$ represents the suspension deformation, $x_{2}=\dot{z}_{s}$ represents the vehicle body vertical velocity, $x_{3}=z_{u}-z_{r}$ represents the tire deformation, and $z_{4}=\dot{z}_{u}$ represents the vertical velocity of the unsprung mass.

$$
\mathbf{A}_{q}=\left[\begin{array}{cccc}
0 & 1 & 0 & -1 \\
-\frac{k_{s}}{m_{s}} & -\frac{c_{p}}{m_{s}} & 0 & \frac{c_{p}}{m_{s}} \\
0 & 0 & 0 & 1 \\
\frac{k_{s}}{m_{u}} & \frac{c_{p}}{m_{u}} & -\frac{k_{t}}{m_{u}} & -\frac{c_{p}}{m_{u}}
\end{array}\right]
$$

$$
\mathbf{B}_{q}=\left[\begin{array}{llll}
0 & 0 & -1 & 0
\end{array}\right]^{T}
$$

\subsubsection{Nonlinear model}

Fig. 2 illustrates the suspension components in a balanced state. $\theta_{0}$ and $\beta_{0}$ respectively denote the initial angle between the lower control arm and horizontal line, and that between the shock absorber and horizontal line. $l_{0}$ represents the initial length of the shock absorber.

When the lower control turns counterclockwise by $\theta^{\circ}$, the displacement of the vehicle body (Point A), lower control arm (Point B), and tire center (Point $\mathrm{C}$ ) relative to the initial balanced positions can be determined. Specifically, the displacements of Points A, B, and C are defined using $\left(y_{A}, z_{A}\right),\left(y_{B}, z_{B}\right)$, and $\left(y_{C}, z_{C}\right)$, respectively, as shown in Eq. (3), where $l_{A}, l_{B}$, and $l_{C}$ respectively indicate the lengths of $\overline{\mathrm{OA}}, \overline{\mathrm{OB}}$, and $\overline{\mathrm{BC}}, \alpha$ represents the angle between $l_{A}$ and the horizontal line, and $\gamma$ is the angle between $l_{C}$ and $l$. When $l_{A}, l_{B}$, and the angles between the lower control arm and horizontal line $\left(\theta_{0}\right.$ and $\theta$ ) are known, the vector loop method can be used to determine the angles between the shock absorber and horizontal line $\left(\beta_{0}\right.$ and $\left.\beta\right)$.

$$
\begin{aligned}
& y_{A}=0 \\
& z_{A}=z_{s} \\
& y_{B}=l_{B}\left(\cos \left(\theta-\theta_{0}\right)-\cos \left(-\theta_{0}\right)\right) \\
& z_{B}=z_{s}+l_{B}\left(\sin \left(\theta-\theta_{0}\right)-\sin \left(-\theta_{0}\right)\right) \\
& y_{C}=l_{C}\left(\cos (\beta-\gamma)-\cos \left(\beta_{0}-\gamma\right)\right)+y_{B} \\
& z_{C}=l_{C}\left(\sin (\beta-\gamma)-\sin \left(\beta_{0}-\gamma\right)\right)+z_{B} \\
& \beta_{0}=\tan ^{-1}\left(\frac{l_{A} \sin \alpha+l_{B} \sin \theta_{0}}{l_{A} \cos \alpha-l_{B} \cos \theta_{0}}\right) \\
& \beta=\tan ^{-1}\left[\frac{l_{A} \sin \alpha+l_{B} \sin \left(\theta_{0}-\theta\right)}{l_{A} \cos \alpha-l_{B} \cos \left(\theta_{0}-\theta\right)}\right]
\end{aligned}
$$

The law of cosines is used to calculate the initial length $\left(l_{0}\right)$ of the shock absorber and the length $(l)$ after the lower control arm turns $\theta$, as shown in Eq. (4). Subsequently, the spring deformation $(\Delta l)$ and the compression rate of the shock absorber spring $(\Delta i)$ are derived, where $\alpha^{\prime}=\alpha+\theta_{0}$.

$$
\begin{aligned}
& l_{0}=\left(l_{A}^{2}+l_{B}^{2}-2 l_{A} l_{B} \cos \alpha^{\prime}\right)^{1 / 2} \\
& l=\left[l_{A}^{2}+l_{B}^{2}-2 l_{A} l_{B} \cos \left(\alpha^{\prime}-\theta\right)\right]^{1 / 2} \\
& (\Delta l)^{2}=\left(l_{0}-l\right)^{2} \\
& \Delta i=i_{0}-i
\end{aligned}
$$

Equation (5) indicates using the Lagrange's Equation to construct the vehicle dynamic model, where $q$ represents the system parameter, $T$ represents the system kinetic energy, $V$ represents the system potential energy, $D$ represents the system dissipating function (e.g., the damping force), and $Q$ represents the generalized force. Substituting the displacements and velocities of the mass points, which are respectively determined in Eq. (3) and 
Eq. (4), into Eq. (5) yields Eq. (6), or the vehicle dynamic function with the vehicle body vertical displacement $\left(z_{s}\right)$ and lower control arm turning angle $(\theta)$ as the system parameters.

$$
\frac{d}{d t} \frac{\partial T}{\partial \dot{q}}+\frac{\partial V}{\partial q}+\frac{\partial D}{\partial \dot{q}}=Q
$$

where,

$$
\begin{aligned}
T= & \frac{1}{2} m_{s} \dot{z}_{s}^{2}+\frac{1}{2} m_{u}\left(\dot{y}_{C}^{2}+\dot{z}_{C}^{2}\right) \\
= & \frac{1}{2}\left(m_{s}+m_{u}\right) \dot{z}_{s}^{2}+\frac{1}{2} m_{u}\left[l_{C}^{2} \beta_{\theta}^{2} \dot{\theta}^{2}+l_{B}^{2} \dot{\theta}^{2}\right. \\
& +2 l_{C} l_{B} \beta_{\theta} \dot{\theta}^{2} \sin (\beta-\gamma) \sin \left(\theta-\theta_{0}\right) \\
& +2 \dot{z}_{s} l_{C} \beta_{\theta} \dot{\theta} \cos (\beta-\gamma)+2 \dot{z}_{s} l_{B} \dot{\theta} \cos \left(\theta-\theta_{0}\right) \\
& \left.+2 l_{C} l_{B} \beta_{\theta} \dot{\theta}^{2} \cos (\beta-\gamma) \cos \left(\theta-\theta_{0}\right)\right] \\
V= & \frac{1}{2} k_{s}(\Delta l)^{2}+\frac{1}{2} k_{t}\left(z_{C}-z_{r}\right)^{2} \\
= & \frac{1}{2} k_{s}\left\{2 a_{1}-b_{1}\left[\cos \alpha^{\prime}+\cos \left(\alpha^{\prime}-\theta\right)\right]\right. \\
& -2\left[a_{1}^{2}-a_{1} b_{1}\left(\cos \alpha^{\prime}+\cos \left(\alpha^{\prime}-\theta\right)\right]\right. \\
& \left.+b_{1}^{2}\left[\cos \alpha^{\prime} \cos \left(\alpha^{\prime}-\theta\right)\right]^{1 / 2}\right\}+\frac{1}{2} k_{t}\left(z_{C}-z_{r}\right)^{2} \\
& =\frac{1}{2} c_{p}(\Delta i)^{2}=\frac{c_{p} b_{1}^{2} \sin ^{2}\left(\alpha^{\prime}-\theta\right) \dot{\theta}^{2}}{8\left[a_{1}-b_{1} \cos \left(\alpha^{\prime}-\theta\right)\right]}
\end{aligned}
$$

where,

$$
\begin{aligned}
& a_{1}=l_{A}^{2}+l_{B}^{2} ; b_{1}=2 l_{A} l_{B} \\
& \beta_{\theta}=\frac{\partial \beta}{\partial \theta}=\frac{l_{A}^{2}-l_{B}^{2}}{2\left[l_{A}^{2}+2 \cos \left(\alpha-\theta_{0}+\theta\right) l_{A} l_{B}+l_{B}^{2}\right]}-\frac{1}{2}
\end{aligned}
$$

When $q=z_{s}$,

$$
\begin{aligned}
& \left(m_{s}+m_{u}\right) \ddot{z}_{s}+\left[m_{u} l_{C} \beta_{\theta} \cos (\beta-\gamma)+m_{u} l_{B} \cos \left(\theta-\theta_{0}\right)\right] \ddot{\theta} \\
& +m_{u} l_{C} \beta_{\theta 2} \dot{\theta}^{2} \cos (\beta-\gamma)-m_{u} l_{C} \beta_{\theta}^{2} \dot{\theta}^{2} \sin (\beta-\gamma) \\
& -m_{u} l_{B} \dot{\theta}^{2} \sin \left(\theta-\theta_{0}\right)+k_{t}\left(z_{C}-z_{r}\right)=0
\end{aligned}
$$

When $q=\theta$,

$\left[m_{u} l_{C}^{2} \beta_{\theta}^{2}+m_{u} l_{\mathrm{B}}^{2}+2 m_{u} l_{C} l_{B} \beta_{\theta} \cos \left(\beta-\gamma-\theta+\theta_{0}\right)\right] \ddot{\theta}$

$+\left[m_{u} l_{C} \beta_{\theta} \cos (\beta-\gamma)+m_{u} l_{B} \cos \left(\theta-\theta_{0}\right)\right] \ddot{z}_{s}$

$+2 m_{u} l_{\mathrm{C}}^{2} \beta_{\theta} \beta_{\theta 2} \dot{\theta}^{2}+2 m_{u} l_{C} l_{B} \beta_{\theta 2} \dot{\theta}^{2} \cos \left(\beta-\gamma-\theta+\theta_{0}\right)$

$-2 m_{u} l_{C} l_{B} \beta_{\theta} \dot{\theta}^{2} \sin \left(\beta-\gamma-\theta+\theta_{0}\right)\left(\beta_{\theta}-1\right)$

$+m_{u} \dot{z}_{s} l_{C} \beta_{\theta 2} \dot{\theta} \cos (\beta-\gamma)-m_{u} \dot{z}_{s} l_{C} \beta_{\theta}^{2} \dot{\theta} \sin (\beta-\gamma)$

$-m_{u} \dot{z}_{s} l_{B} \dot{\theta} \sin \left(\theta-\theta_{0}\right)+\frac{c_{p} b_{1}^{2} \sin ^{2}\left(\alpha^{\prime}-\theta\right) \dot{\theta}}{4\left[a_{1}-b_{1} \cos \left(\alpha^{\prime}-\theta\right)\right]}$

$+k_{t}\left(z_{C}-z_{r}\right)\left[l_{C} \cos (\beta-\gamma) \beta_{\theta}+l_{B} \cos \left(\theta-\theta_{0}\right)\right]$

$-\frac{1}{2} k_{s} \sin \left(\alpha^{\prime}-\theta\right)\left[b_{1}+\frac{d_{1}}{\left[c_{1}-d_{1} \cos \left(\alpha^{\prime}-\theta\right)\right]^{1 / 2}}\right]=0$

Where, $c_{1}=a_{1}^{2}-a_{1} b_{1} \cos \alpha^{\prime} ; d_{1}=a_{1} b_{1}-b_{1}^{2} \cos \alpha^{\prime}$

$\beta_{\theta \theta}=\frac{d \beta_{\theta}}{d \theta}=\frac{l_{A} l_{B} \sin \left(\alpha-\theta_{0}+\theta\right)\left(l_{A}^{2}-l_{B}^{2}\right)}{\left[l_{A}^{2}+2 l_{A} l_{B} \cos \left(\alpha-\theta_{0}+\theta\right)+l_{B}^{2}\right]^{2}}$

The state vector of the dynamic function of the nonlinear suspension model is $x=\left[\begin{array}{llll}x_{1} & x_{2} & x_{3} & x_{4}\end{array}\right]$ $=\left[\begin{array}{llll}z_{s} & \dot{z}_{s} & \theta & \dot{\theta}\end{array}\right]^{T}$, and the state functions are expressed as Eq. (7).

$$
\begin{aligned}
& \dot{x}_{1}=x_{2} \\
& \dot{x}_{2}=f_{1}\left(x_{1}, x_{2}, x_{3}, x_{4}, z_{r}\right) \\
& \dot{x}_{3}=x_{4} \\
& \dot{x}_{4}=f_{2}\left(x_{1}, x_{2}, x_{3}, x_{4}, z_{r}\right)
\end{aligned}
$$

where,

$$
\begin{aligned}
f_{1}= & \frac{-1}{g_{1} g_{3}-g_{2} g_{4}}\left[g_{3} g_{5}+k_{t}\left(z_{C}-z_{r}\right)\left(g_{3}-g_{2} g_{6}\right)-g_{2} g_{7}\right] \\
f_{2}= & \frac{-1}{g_{2} g_{4}-g_{1} g_{3}}\left[g_{4} g_{5}+k_{t}\left(z_{C}-z_{r}\right)\left(g_{4}-g_{1} g_{6}\right)-g_{1} g_{7}\right] \\
g_{1}= & \left(m_{s}+m_{u}\right) \\
g_{2}= & \left(m_{u} l_{C} \beta_{\theta} \cos (\beta-\gamma)+m_{u} l_{B} \cos \left(\theta-\theta_{0}\right)\right) \\
g_{3}= & m_{u} l_{C}^{2} \beta_{\theta}^{2}+m_{u} l_{\mathrm{B}}^{2}+2 m_{u} l_{C} l_{B} \beta_{\theta} \cos \left(\beta-\gamma-\theta+\theta_{0}\right) \\
g_{4}= & m_{u} l_{C} \beta_{\theta} \cos (\beta-\gamma)+m_{u} l_{B} \cos \left(\theta-\theta_{0}\right) \\
g_{5}= & m_{u} l_{C} \beta_{\theta 2} \dot{\theta}^{2} \cos (\beta-\gamma)-m_{u} l_{C} \beta_{\theta}^{2} \dot{\theta}^{2} \sin (\beta-\gamma) \\
& -m_{u} l_{B} \dot{\theta}^{2} \sin \left(\theta-\theta_{0}\right) \\
g_{6}= & l_{C} \cos (\beta-\gamma) \beta_{\theta}+l_{B} \cos \left(\theta-\theta_{0}\right) \\
g_{7}= & 2 m_{u} l_{\mathrm{C}}^{2} \beta_{\theta} \beta_{\theta 2} \dot{\theta}^{2}+2 m_{u} l_{C} l_{B} \beta_{\theta 2} \dot{\theta}^{2} \cos \left(\beta-\gamma-\theta+\theta_{0}\right) \\
& -2 m_{u} l_{C} l_{B} \beta_{\theta} \dot{\theta}^{2} \sin \left(\beta-\gamma-\theta+\theta_{0}\right)\left(\beta_{\theta}-1\right) \\
& +m_{u} \dot{z}_{s} l_{C} \beta_{\theta 2} \dot{\theta} \cos (\beta-\gamma)-m_{u} \dot{z}_{s} l_{C} \beta_{\theta}^{2} \dot{\theta} \sin (\beta-\gamma) \\
& -m_{u} \dot{z}_{s} l_{B} \dot{\theta} \sin \left(\theta-\theta_{0}\right)+\frac{c_{p} b_{1}^{2} \sin ^{2}\left(\alpha^{\prime}-\theta\right) \dot{\theta}}{4\left[a_{1}-b_{1} \cos \left(\alpha^{\prime}-\theta\right)\right]} \\
& -\frac{1}{2} k_{s} \sin \left(\alpha^{\prime}-\theta\right)\left[b_{1}-\frac{d_{1}}{\left[c_{1}-d_{1} \cos \left(\alpha^{\prime}-\theta\right)\right]^{1 / 2}}\right]
\end{aligned}
$$

\subsubsection{Linearization of the nonlinear model}

A nonlinear model in a balanced state can be linearized, where the balanced state is expressed as $x_{e}=\left(x_{1 e}, x_{2 e}, x_{3 e}, x_{4 e}\right)=(0,0,0,0)$. The linearized state function is displayed as Eq. (8). Because the linearized function is overly long, the matrix elements expressed as partial differential equations are not displayed herein to conform to the space limitation of this manuscript.

$$
\dot{x}=\mathbf{A}_{q l} x+\mathbf{B}_{q l} \dot{z}_{r}
$$




$$
\begin{aligned}
\mathbf{A}_{q l} & =\left[\begin{array}{cccc}
0 & 1 & 0 & 0 \\
\frac{\partial f_{1}}{\partial x_{1}} & \frac{\partial f_{1}}{\partial x_{2}} & \frac{\partial f_{1}}{\partial x_{3}} & \frac{\partial f_{1}}{\partial x_{4}} \\
0 & 0 & 0 & 1 \\
\frac{\partial f_{2}}{\partial x_{1}} & \frac{\partial f_{2}}{\partial x_{2}} & \frac{\partial f_{2}}{\partial x_{3}} & \frac{\partial f_{2}}{\partial x_{4}}
\end{array}\right]_{x=x_{e}} \\
\mathbf{B}_{q l} & =\left[\begin{array}{llll}
0 & \frac{\partial f_{1}}{\partial z_{r}} & 0 & \frac{\partial f_{2}}{\partial z_{r}}
\end{array}\right]_{z_{r}=0}^{T}
\end{aligned}
$$

\subsubsection{Adams 1/4 vehicle suspension model}

Fig. 3 depicts a 1/4 vehicle suspension model constructed using the ADAMS-View module. This model serves as the reference to be compared with other models.

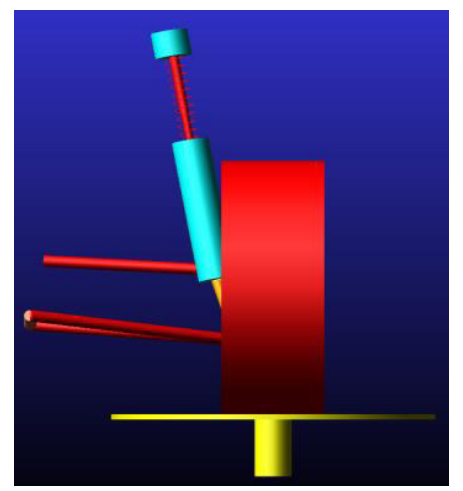

Fig. 3. ADAMS 1/4 vehicle suspension model.

\subsection{The $1 / 2$ vehicle suspension models}

$1 / 2$ vehicle suspension models are more informative than their 1/4 counterparts. As shown in Fig. 4, data such as the vertical displacement $\left(z_{s}\right)$ at the vehicle center of mass and the pitch angle $(\phi)$ are also available in a $1 / 2$ model.

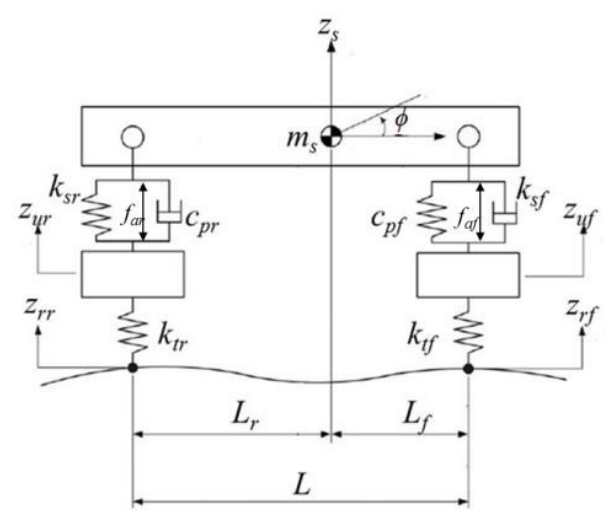

Fig. 4. 1/2 vehicle suspension model.

\subsubsection{Traditional suspension model}

Dynamic equations for determining $z_{s}$ and $\phi$ are expressed as Eq. (9) and Eq. (10), respectively, where $F_{f}$ and $F_{r}$ respectively denote the reaction forces that the front and rear axle suspensions exert on the vehicle body; $L_{f}$ and $L_{r}$ respectively indicate the horizontal distances of the vehicle center of mass to the front and rear axles; $L$ is the distance between the front and rear axles; $I_{P}$ represents the inertia of mass moment in the pitch direction of the vehicle body; and $z_{s f}$ and $z_{s r}$ respectively denote the vertical displacements of the front and rear axle sprung mass when the rear tires bounce because of an uneven road surface. Equations (9) and (10) yield the dynamic vertical accelerations of the front and rear axle sprung mass $\left(\ddot{z}_{s f}\right.$ and $\ddot{z}_{s r}$ ), as shown in Eq. (11). The vertical accelerations of the front and rear axle unsprung mass $\ddot{z}_{u f}$ and $\ddot{z}_{u r}$ are calculated using the same expressions for the $1 / 4$ vehicle suspension model, as shown in Eq. (12). Equations (11) and (12) can be rearranged into a matrix form, as shown in Eq. (13), where $x=\left[\begin{array}{llllllll}x_{1} & x_{2} & x_{3} & x_{4} & x_{5} & x_{6} & x_{7} & x_{8}\end{array}\right]^{T}$ is the $1 / 2$ vehicle state vector; $x_{1}=z_{s f}-z_{u f}$ is the deformation of the front axle suspension; $x_{2}=\dot{z}_{s f}$ is the vertical velocity of the front axle sprung mass; $x_{3}=z_{u f}-z_{r f}$ is the deformation of the front axle tire; $x_{4}=\dot{z}_{u f}$ is the vertical velocity of the front axle unsprung mass; $x_{5}=z_{s r}-z_{u r}$ is the deformation of the rear axle suspension; $x_{6}=\dot{z}_{s r}$ is the vertical velocity of the rear axle sprung mass; $x_{7}=z_{u r}-z_{r r}$ is the deformation of the rear axle tire; and $z_{8}=\dot{z}_{u r}$ is the vertical velocity of the rear axle unsprung mass. Finally, $\dot{z}_{h r}=\left[\begin{array}{cc}\dot{z}_{r f} & \dot{z}_{r r}\end{array}\right]$ represents the road surface input, and $f_{h a}=\left[\begin{array}{ll}f_{a f} & f_{a r}\end{array}\right]$ denotes the driving force input.

$$
\begin{aligned}
& m_{s} \ddot{z}_{s}=\left[-k_{s f}\left(z_{s f}-z_{u f}\right)-c_{p f}\left(\dot{z}_{s f}-\dot{z}_{u f}\right)+f_{a f}\right] \\
& +\left[-k_{s r}\left(z_{s r}-z_{u r}\right)-c_{p r}\left(\dot{z}_{s r}-\dot{z}_{u r}\right)+f_{a r}\right] \\
& =F_{f}+F_{r} \\
& \begin{aligned}
I_{p} \ddot{\phi} & =\left[-k_{s r}\left(z_{s r}-z_{u r}\right)-c_{p r}\left(\dot{z}_{s r}-\dot{z}_{u r}\right)+f_{a r}\right] L_{r} \\
& -\left[-k_{s f}\left(z_{s f}-z_{u f}\right)-c_{p f}\left(\dot{z}_{s f}-\dot{z}_{u f}\right)+f_{a f}\right] L_{f}
\end{aligned} \\
& =F_{r} L_{r}-F_{f} L_{f} \\
& z_{s}=\frac{L_{r} z_{s f}+L_{f} z_{s r}}{L} \\
& \phi=\frac{-z_{s f}+z_{s r}}{L} \\
& \ddot{z}_{s f}=\left(\frac{1}{m_{s}}-\frac{L_{f} L_{r}}{I_{p}}\right) F_{r}+\left(\frac{1}{m_{s}}+\frac{L_{f}^{2}}{I_{p}}\right) F_{f} \\
& \ddot{z}_{s r}=\left(\frac{1}{m_{s}}+\frac{L_{r}^{2}}{I_{p}}\right) F_{r}+\left(\frac{1}{m_{s}}-\frac{L_{f} L_{r}}{I_{p}}\right) F_{f} \\
& m_{u f} \ddot{z}_{u f}=k_{s f}\left(z_{s f}-z_{u f}\right)+c_{p f}\left(\dot{z}_{s f}-\dot{z}_{u f}\right) \\
& -k_{t f}\left(z_{u f}-z_{r f}\right)-f_{a f} \\
& m_{u r} \ddot{z}_{u r}=k_{s r}\left(z_{s r}-z_{u r}\right)+c_{p r}\left(\dot{z}_{s r}-\dot{z}_{u r}\right) \\
& -k_{t r}\left(z_{u r}-z_{r r}\right)-f_{a r} \\
& \dot{x}=\mathbf{A}_{h} x+\mathbf{B}_{h z} \dot{z}_{h r}+\mathbf{B}_{h f} f_{h a}
\end{aligned}
$$




$$
\begin{aligned}
\mathbf{A}_{h} & =\left[\begin{array}{ll}
\mathbf{A}_{h a} & \mathbf{A}_{h b} \\
\mathbf{A}_{h c} & \mathbf{A}_{h d}
\end{array}\right] \\
\mathbf{B}_{h z} & =\left[\begin{array}{cccccccc}
0 & 0 & -1 & 0 & 0 & 0 & 0 & 0 \\
0 & 0 & 0 & 0 & 0 & 0 & -1 & 0
\end{array}\right]^{T} \\
\mathbf{B}_{h f} & =\left[\begin{array}{ll}
\mathbf{B}_{h f a} & \mathbf{B}_{h f b}
\end{array}\right]^{T}
\end{aligned}
$$

Where,

$$
\begin{aligned}
& \mathbf{A}_{h a}=\left[\begin{array}{cccc}
0 & 0 & 1 & -1 \\
-k_{s f}\left(\frac{1}{m_{s}}+\frac{L_{f}^{2}}{I_{p}}\right) & -c_{p f}\left(\frac{1}{m_{s}}+\frac{L_{f}^{2}}{I_{p}}\right) & 0 & c_{p f}\left(\frac{1}{m_{s}}+\frac{L_{f}^{2}}{I_{p}}\right) \\
0 & 0 & 0 & 1 \\
\frac{k_{s f}}{m_{u f}} & \frac{c_{p f}}{m_{u f}} & -\frac{k_{t f}}{m_{u f}} & -\frac{c_{p f}}{m_{u f}}
\end{array}\right] \\
& \mathbf{A}_{h b}=\left[\begin{array}{ccccc}
0 & 0 & 0 & 0 \\
-k_{s r}\left(\frac{1}{m_{s}}-\frac{L_{f} L_{r}}{I_{p}}\right) & -c_{p r}\left(\frac{1}{m_{s}}-\frac{L_{f} L_{r}}{I_{p}}\right) & 0 & c_{p r}\left(\frac{1}{m_{s}}-\frac{L_{f} L_{r}}{I_{p}}\right) \\
0 & 0 & 0 & 0 \\
0 & 0 & 0 & 0
\end{array}\right] \\
& \mathbf{A}_{h c}=\left[\begin{array}{ccccc}
0 & 0 & 0 & 0 \\
-k_{s f}\left(\frac{1}{m_{s}}-\frac{L_{f} L_{r}}{I_{p}}\right) & -c_{p f}\left(\frac{1}{m_{s}}-\frac{L_{f} L_{r}}{I_{p}}\right) & 0 & c_{p f}\left(\frac{1}{m_{s}}-\frac{L_{f} L_{r}}{I_{p}}\right) \\
0 & 0 & 0 & 0 \\
0 & 0 & 0 & 0
\end{array}\right]
\end{aligned}
$$

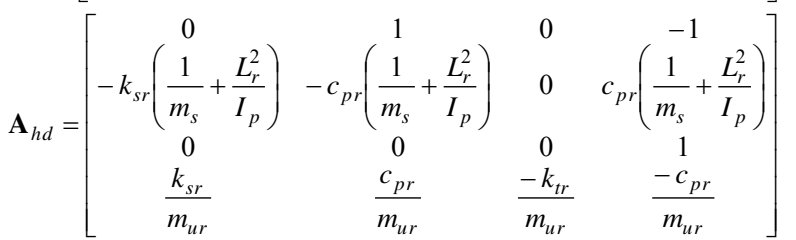

$$
\begin{aligned}
& \mathbf{B}_{h f a}=\left[\begin{array}{cccc}
0 & \left(\frac{1}{m_{s}}+\frac{L_{f}^{2}}{I_{p}}\right) & \frac{-1}{m_{u f}} & 0 \\
0 & \left(\frac{1}{m_{s}}-\frac{L_{f} L_{r}}{I_{p}}\right) & 0 & 0
\end{array}\right]
\end{aligned}
$$

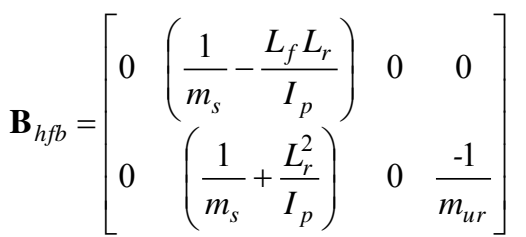

\subsubsection{Nonlinear model}

Similar to the $1 / 4$ nonlinear model, the $1 / 2$ nonlinear model also adopts Eq. (3) and Eq. (4) to determine the displacements and velocities of the sprung and unsprung mass in the front and rear axles as well as the spring deformation and compression rate of the shock absorber. $\dot{z}_{s}$ and $\dot{\phi}$ are determined using Eq. (10) and then substituted into the kinetic energy, potential energy, and dissipating functions, as expressed in Eq. (14) .

$$
\begin{aligned}
T & =\frac{1}{2} m_{s} \dot{z}_{s}^{2}+\frac{1}{2} I_{p} \dot{\phi}^{2}+\frac{1}{2} m_{u r}\left(\dot{y}_{C r}^{2}+\dot{z}_{C r}^{2}\right) \\
& +\frac{1}{2} m_{u f}\left(\dot{y}_{C f}^{2}+\dot{z}_{C f}^{2}\right) \\
V & =\frac{1}{2} k_{s r}\left(\Delta l_{r}\right)^{2}+\frac{1}{2} k_{t r}\left(z_{C r}-z_{r r}\right)^{2}+\frac{1}{2} k_{s f}\left(\Delta l_{f}\right)^{2} \\
& +\frac{1}{2} k_{t f}\left(z_{C f}-z_{r f}\right)^{2} \\
D & =\frac{1}{2} c_{p r}\left(\Delta i_{r}\right)^{2}+\frac{1}{2} c_{p f}\left(\Delta \dot{l}_{f}\right)^{2}
\end{aligned}
$$

The Lagrange's Equation, Eq. (5), is used to construct the vehicle dynamic motion with the vertical displacements of the front and rear axle sprung mass $\left(z_{s f}\right.$ and $\left.z_{s r}\right)$ and lower control arm rotation $\left(\theta_{f}\right.$ and $\left.\theta_{r}\right)$ as the system parameters, as shown in Eq. (15). Solving Eq. (15) determines the functional relationship of $\ddot{z}_{s f}$, $\ddot{z}_{s r}, \ddot{\theta}_{f}$, and $\ddot{\theta}_{r}$ with the system states. The state function of the nonlinear model is expressed as Eq. (16), where the state vector $x=\left[\begin{array}{llllllll}x_{1} & x_{2} & x_{3} & x_{4} & x_{5} & x_{6} & x_{7} & x_{8}\end{array}\right]^{T}=$ $\left[\begin{array}{cccccccc}z_{s f} & \dot{z}_{s f} & \theta_{f} & \dot{\theta}_{f} & z_{s r} & \dot{z}_{s r .} & \theta_{r} & \dot{\theta}_{r}\end{array}\right]$, $f_{3}=\ddot{z}_{s f}, f_{4}=\ddot{\theta}_{f}, f_{5}=\ddot{z}_{s r}$, and $f_{6}=\ddot{\theta}_{r}$.

When $q=z_{s f}$,

$\frac{1}{L^{2}}\left(m_{s} L_{r}^{2}+I_{p}+m_{u f} L^{2}\right) \ddot{z}_{s f}+\frac{1}{L^{2}}\left(m_{s} L_{f} L_{r}\right.$

$\left.-I_{p}\right) \ddot{z}_{s r}+\left[m_{u f} l_{C f} \beta_{\theta f} \cos \left(\beta_{f}-\gamma_{f}\right)\right.$

$\left.+m_{u f} l_{B f} \cos \left(\theta_{f}-\theta_{0 f}\right)\right] \ddot{\theta}_{f}$

$+m_{u f} l_{C f} \beta_{\theta 2 f} \dot{\theta}_{f}^{2} \cos \left(\beta_{f}-\gamma_{f}\right)$

$-m_{u f} l_{C f} \beta_{\theta f}^{2} \dot{\theta}_{f}^{2} \sin \left(\beta_{f}-\gamma_{f}\right)$

$-m_{u f} l_{B f} \dot{\theta}_{f}^{2} \sin \left(\theta_{f}-\theta_{0 f}\right)+k_{t f}\left(z_{C f}-z_{r f}\right)=0$

When $q=\theta_{f}$

$\left[m_{u f} l_{C f}^{2} \beta_{\theta f}^{2}+m_{u f} l_{B f}^{2}+2 m_{u f} l_{C f} l_{B f} \beta_{\theta f} \cos \left(\beta_{f}\right.\right.$

$\left.\left.-\gamma_{f}-\theta_{f}+\theta_{0 f}\right)\right] \ddot{\theta}_{f}+\left[m_{u f} l_{C f} \beta_{\theta f} \cos \left(\beta_{f}-\gamma_{f}\right)\right.$

$\left.+m_{u f} l_{B f} \cos \left(\theta_{f}-\theta_{0 f}\right)\right] \ddot{z}_{s f}+2 m_{u f} l_{\mathrm{Cf}}^{2} \beta_{\theta f} \beta_{\theta 2 \mathrm{f}} \dot{\theta}_{f}^{2}$

$+2 m_{u f} l_{C f} l_{B f} \beta_{\theta 2 \mathrm{f}} \dot{\theta}_{f}^{2} \cos \left(\beta_{f}-\gamma_{f}-\theta_{f}+\theta_{0 f}\right)$

$-2 m_{u f} l_{C f} l_{B f} \beta_{\theta f} \dot{\theta}_{f}^{2} \sin \left(\beta_{f}-\gamma_{f}-\theta_{f}+\theta_{0 f}\right)\left(\beta_{\theta f}\right.$

$-1)+m_{u f} \dot{z}_{s f} l_{C f} \beta_{\theta 2 f} \dot{\theta}_{f} \cos \left(\beta_{f}-\gamma_{f}\right)$

$-m_{u f} \dot{z}_{s f} l_{C f} \beta_{\theta f}^{2} \dot{\theta}_{f} \sin \left(\beta_{f}-\gamma_{f}\right)$

$-m_{u f} \dot{z}_{s f} l_{B f} \dot{\theta}_{f} \sin \left(\theta_{f}-\theta_{0 f}\right)$

$+\frac{c_{p f} b_{1 f}^{2} \sin ^{2}\left(\alpha_{f}^{\prime}-\theta_{f}\right) \dot{\theta}_{f}}{4\left[a_{1 f}-b_{1 f} \cos \left(\alpha_{f}^{\prime}-\theta_{f}\right)\right]}$

$+k_{t f}\left(z_{C f}-z_{r f}\right) l_{C f} \cos \left(\beta_{f}-\gamma_{f}\right) \beta_{\theta f}+l_{B f} \cos \left(\theta_{f}\right.$

$\left.\left.-\theta_{0 f}\right)\right]-\frac{1}{2} k_{s f} \sin \left(\alpha_{f}^{\prime}-\theta_{f}\right)\left[b_{1 f}\right.$

$\left.-\frac{d_{1 f}}{\left[c_{1 f}-d_{1 f} \cos \left(\alpha_{f}^{\prime}-\theta_{f}\right)\right]^{1 / 2}}\right]+l_{B f} f_{a f}=0$ 
When $q=z_{s r}$,

$\frac{1}{L^{2}}\left(m_{s} L_{f}^{2}+I_{p}+m_{u r} L^{2}\right) \ddot{z}_{s r}+\frac{1}{L^{2}}\left(m_{s} L_{f} L_{r}\right.$

$\left.-I_{p}\right) \ddot{z}_{s f}+\left[m_{u r} l_{C r} \beta_{\theta r} \cos \left(\beta_{r}-\gamma_{r}\right)\right.$

$\left.+m_{u r} l_{B r} \cos \left(\theta_{r}-\theta_{0 r}\right)\right] \ddot{\theta}_{r}$

$+m_{u r} l_{C r} \beta_{\theta 2 \mathrm{r}} \dot{\theta}_{r}^{2} \cos \left(\beta_{r}-\gamma_{r}\right)$

$-m_{u r} l_{C r} \beta_{\theta r}^{2} \dot{\theta}_{r}^{2} \sin \left(\beta_{r}-\gamma_{r}\right)$

$-m_{u r} l_{B r} \dot{\theta}_{r}^{2} \sin \left(\theta_{r}-\theta_{0 r}\right)+k_{t r}\left(z_{C r}-z_{r r}\right)=0$

When $q=\theta_{r}$,

$\left[m_{u r} l_{C r}^{2} \beta_{\theta r}^{2}+m_{u r} l_{B r}^{2}+2 m_{u r} l_{C r} l_{B r} \beta_{\theta r} \cos \left(\beta_{r}\right.\right.$

$\left.\left.-\gamma_{r}-\theta_{r}+\theta_{0 r}\right)\right] \ddot{\theta}_{r}+\left[m_{u r} l_{C r} \beta_{\theta r} \cos \left(\beta_{r}-\gamma_{r}\right)\right.$

$\left.+m_{u r} l_{B r} \cos \left(\theta_{r}-\theta_{0 r}\right)\right] \ddot{z}_{s r}+2 m_{u r} l_{\mathrm{Cr}}^{2} \beta_{\theta r} \beta_{\theta 2 \mathrm{r}} \dot{\theta}_{r}^{2}$

$+2 m_{u r} l_{C r} l_{B r} \beta_{\theta 2 \mathrm{r}} \dot{\theta}_{r}^{2} \cos \left(\beta_{r}-\gamma_{r}-\theta_{r}+\theta_{0 r}\right)$

$-2 m_{u r} l_{C r} l_{B r} \beta_{\theta r} \dot{\theta}_{r}^{2} \sin \left(\beta_{r}-\gamma_{r}-\theta_{r}+\theta_{0 r}\right)\left(\beta_{\theta r}-1\right)$

$+m_{u r} \dot{z}_{s r} l_{C r} \beta_{\theta 2 r} \dot{\theta}_{r} \cos \left(\beta_{r}-\gamma_{r}\right)$

$-m_{u r} \dot{z}_{s r} l_{C r} \beta_{\theta r}^{2} \dot{\theta}_{r} \sin \left(\beta_{r}-\gamma_{r}\right)-m_{u r} \dot{z}_{s r} l_{B r} \dot{\theta}_{r} \sin \left(\theta_{r}\right.$

$\left.-\theta_{0 r}\right)+\frac{c_{p r} b_{1 r}^{2} \sin ^{2}\left(\alpha_{r}^{\prime}-\theta_{r}\right) \dot{\theta}_{r}}{4\left[a_{1 r}-b_{1 r} \cos \left(\alpha_{r}^{\prime}-\theta_{r}\right)\right]}$

$+k_{t r}\left(z_{C r}-z_{r r}\right)\left[l_{C r} \cos \left(\beta_{r}-\gamma_{r}\right) \beta_{\theta r}+l_{B r} \cos \left(\theta_{r}\right.\right.$

$\left.\left.-\theta_{0 r}\right)\right]-\frac{1}{2} k_{s r} \sin \left(\alpha_{r}^{\prime}-\theta_{r}\right)\left[b_{1 r}\right.$

$\left.-\frac{d_{1 r}}{\left[c_{1 r}-d_{1 r} \cos \left(\alpha_{r}^{\prime}-\theta_{r}\right)\right]^{1 / 2}}\right]+l_{B r} f_{a r}=0$

$\dot{x}_{1}=x_{2}$

$\dot{x}_{2}=f_{3}\left(x_{1}, x_{2}, x_{3}, x_{4}, x_{5}, x_{6}, x_{7}, x_{8}, z_{r f}, z_{r r}, f_{a f}, f_{a r}\right)$

$\dot{x}_{3}=x_{4}$

$\dot{x}_{4}=f_{4}\left(x_{1}, x_{2}, x_{3}, x_{4}, x_{5}, x_{6}, x_{7}, x_{8}, z_{r f}, z_{r r}, f_{a f}, f_{a r}\right)$

$\dot{x}_{5}=x_{6}$

$\dot{x}_{6}=f_{5}\left(x_{1}, x_{2}, x_{3}, x_{4}, x_{5}, x_{6}, x_{7}, x_{8}, z_{r f}, z_{r r}, f_{a f}, f_{a r}\right)$

$\dot{x}_{7}=x_{8}$

$\dot{x}_{8}=f_{6}\left(x_{1}, x_{2}, x_{3}, x_{4}, x_{5}, x_{6}, x_{7}, x_{8}, z_{r f}, z_{r r}, f_{a f}, f_{a r}\right)$

\subsubsection{Linearization of the nonlinear model}

The nonlinear model in the balanced state is linearized, with the balanced state expressed as $x_{e}=\left(x_{1 e}, x_{2 e}, x_{3 e}, x_{4 e}, x_{5 e}, x_{6 e}, x_{7 e}, x_{8 e}\right)=(0,0,0,0,0,0,0,0)$. Equation (17) yields the state function of the linearized model.

$$
\dot{x}=\mathbf{A}_{h l} x+\mathbf{B}_{h l f} z_{r f}+\mathbf{B}_{h l r} z_{r r}+\mathbf{B}_{h l f f} f_{a f}+\mathbf{B}_{h l f r} f_{a r}
$$

$$
\begin{aligned}
& {\left[\begin{array}{cccccccc}
0 & 1 & 0 & 0 & 0 & 0 & 0 & 0 \\
\frac{\partial f_{3}}{\partial x_{1}} & \frac{\partial f_{3}}{\partial x_{2}} & \frac{\partial f_{3}}{\partial x_{3}} & \frac{\partial f_{3}}{\partial x_{4}} & \frac{\partial f_{3}}{\partial x_{5}} & \frac{\partial f_{3}}{\partial x_{6}} & \frac{\partial f_{3}}{\partial x_{7}} & \frac{\partial f_{3}}{\partial x_{8}}
\end{array}\right.} \\
& \mathbf{A}_{h l}=\mid \begin{array}{cccccccc}
0 & 0 & 0 & 1 & 0 & 0 & 0 & 0 \\
\frac{\partial f_{4}}{\partial x_{1}} & \frac{\partial f_{4}}{\partial x_{2}} & \frac{\partial f_{4}}{\partial x_{3}} & \frac{\partial f_{4}}{\partial x_{4}} & \frac{\partial f_{4}}{\partial x_{5}} & \frac{\partial f_{4}}{\partial x_{6}} & \frac{\partial f_{4}}{\partial x_{7}} & \frac{\partial f_{4}}{\partial x_{8}} \\
0 & 0 & 0 & 0 & 0 & 1 & 0 & 0 \\
\frac{\partial f_{5}}{\partial x_{1}} & \frac{\partial f_{5}}{\partial x_{2}} & \frac{\partial f_{5}}{\partial x_{3}} & \frac{\partial f_{5}}{\partial x_{4}} & \frac{\partial f_{5}}{\partial x_{5}} & \frac{\partial f_{5}}{\partial x_{6}} & \frac{\partial f_{5}}{\partial x_{7}} & \frac{\partial f_{5}}{\partial x_{8}} \\
0 & 0 & 0 & 0 & 0 & 0 & 0 & 1
\end{array} \\
& {\left[\begin{array}{cccccccc}
0 & 0 & 0 & 0 & 0 & 0 & 0 & 1 \\
\frac{\partial f_{6}}{\partial x_{1}} & \frac{\partial f_{6}}{\partial x_{2}} & \frac{\partial f_{6}}{\partial x_{3}} & \frac{\partial f_{6}}{\partial x_{4}} & \frac{\partial f_{6}}{\partial x_{5}} & \frac{\partial f_{6}}{\partial x_{6}} & \frac{\partial f_{6}}{\partial x_{7}} & \frac{\partial f_{6}}{\partial x_{8}}
\end{array}\right]_{x=x_{c}}} \\
& \mathbf{B}_{h l f}=\left[\begin{array}{llllllll}
0 & \frac{\partial f_{3}}{\partial z_{r f}} & 0 & \frac{\partial f_{4}}{\partial z_{r f}} & 0 & \frac{\partial f_{5}}{\partial z_{r f}} & 0 & \frac{\partial f_{6}}{\partial z_{r f}}
\end{array}\right]_{z_{r f}=0} \\
& \mathbf{B}_{h l r}=\left[\begin{array}{llllllll}
0 & \frac{\partial f_{3}}{\partial z_{r r}} & 0 & \frac{\partial f_{4}}{\partial z_{r r}} & 0 & \frac{\partial f_{5}}{\partial z_{r r}} & 0 & \frac{\partial f_{6}}{\partial z_{r r}}
\end{array}\right]_{z_{r r}=0} \\
& \mathbf{B}_{\text {hlff }}=\left[\begin{array}{llllllll}
0 & \frac{\partial f_{3}}{\partial f_{a r}} & 0 & \frac{\partial f_{4}}{\partial f_{a r}} & 0 & \frac{\partial f_{5}}{\partial f_{a r}} & 0 & \frac{\partial f_{6}}{\partial f_{a r}}
\end{array}\right]_{f_{a f}=0} \\
& \mathbf{B}_{h l f r}=\left[\begin{array}{llllllll}
0 & \frac{\partial f_{3}}{\partial f_{a r}} & 0 & \frac{\partial f_{4}}{\partial f_{a r}} & 0 & \frac{\partial f_{5}}{\partial f_{a r}} & 0 & \frac{\partial f_{6}}{\partial f_{a r}}
\end{array}\right]_{f_{a r}=0}
\end{aligned}
$$

\subsubsection{Adams full vehicle suspension model}

Fig. 5 illustrates the full vehicle suspension model built with the ADAMS-Car module. This model serves as the reference to be compared with the other models.

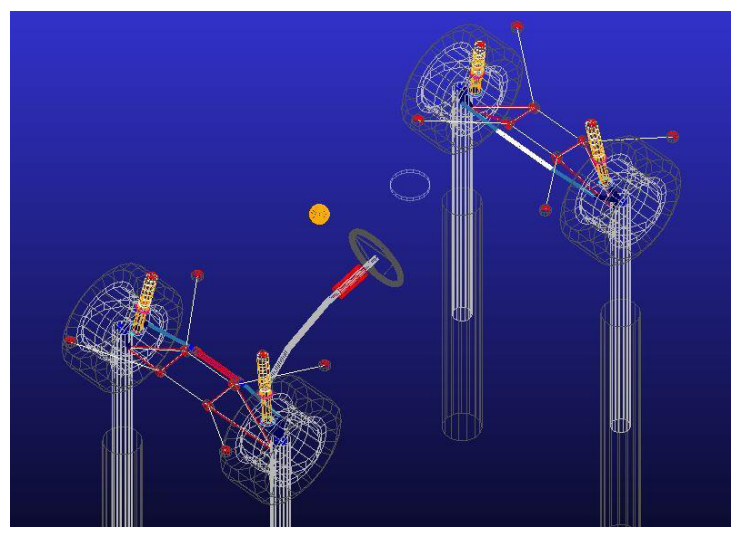

Fig. 5. ADAMS-Car full vehicle suspension model.

\section{Initial balanced positions of suspension components}

The nonlinear model of suspension geometry induces distinct system responses (nonlinear behavior) under dissimilar swing positions of the lower control arm. Therefore, the initial positions of a loaded suspension system must be identified prior to determining the system responses accurately.

In this study, a static balance analysis was conducted (Fig. 6), in conjunction with with a kinematics analysis performed using the vector loop method (Fig. 5)[8], to 
determine the initial angle of the lower arm $\left(\theta_{0}\right)$ under various loads. $F_{W}$ represents the force that the road surface exerts on the suspension (tires), $F_{B}$ represents the reaction force that the lower control arm exerts on the suspension along the $\overline{\mathrm{AB}}$ direction, and $F_{C}$ represents the force that the shock absorber spring exerts on the suspension. The functional relationships of the static balance functions and kinematics functions are expressed as (18) and (19).
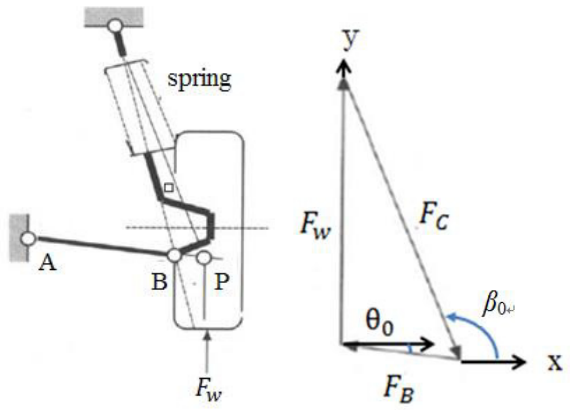

Fig. 6. Static balance analysis of the suspension system.

$$
\begin{gathered}
F_{W}+F_{C}+F_{B}=0 \\
F_{x}=-F_{B} \cos \left(-\theta_{0}\right)-F_{C} \cos \beta_{0}=0 \\
F_{y}=-F_{B} \sin \left(-\theta_{0}\right)-F_{C} \sin \beta_{0}=F_{W} \\
\theta_{0}=\cos ^{-1}\left(\frac{l_{A}^{2}+l_{B}^{2}-r_{3}^{2}}{2 l_{A} l_{B}}\right)-\alpha \\
\beta_{0}=\tan ^{-1}\left(\frac{l_{A} \sin \alpha+l_{B} \sin \theta_{0}}{l_{A} \cos \alpha-l_{B} \cos \theta_{0}}\right)
\end{gathered}
$$

\section{Simulation and discussions}

\subsection{Basic suspension parameters}

The parameter settings are described as follows: front and rear sprung mass $\left(m_{s f}=143 \mathrm{~kg}, m_{s r}=90 \mathrm{~kg}\right)$, front and rear tire mass $\left(m_{u f}=m_{u r}=10 \mathrm{~kg}\right)$, stiffness $\left(k_{t f}=k_{t r}=\right.$ $200 \mathrm{kN} / \mathrm{m})$; interaxle distance $(L=1.8 \mathrm{~m})$, and inertia of mass moment in the vehicle body pitch direction $\left(I_{P}=\right.$ $1100 \mathrm{~kg} \cdot \mathrm{m}^{2}$ ). Fig. 7 depicts the spring stiffness; the springs were compressed by $10 \mathrm{~mm}$ when installed. Fig. 8 illustrates the damping characteristics of the shock absorbers. Suspension geometry was configured according to Fig. 2 and the parameters defined in Section 2.1.2: $l_{A}=0.545 \mathrm{~m}, l_{B}=0.336 \mathrm{~m}, l_{C}=0.107 \mathrm{~m}, \alpha=$ $65.965^{\circ}$, and $\gamma=36.15^{\circ}$; before the system was loaded, the parameters were set as $l_{0}=0.5554 \mathrm{~m}, \beta_{0}=101.52^{\circ}$, and $\theta_{0}=-7.964^{\circ}$.

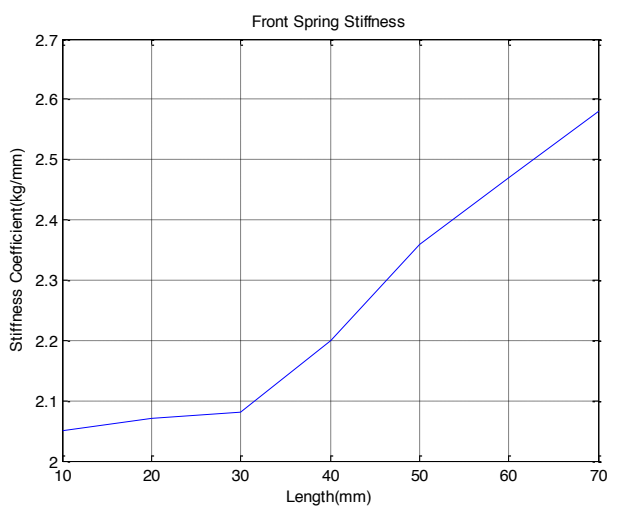

(a) Front axle

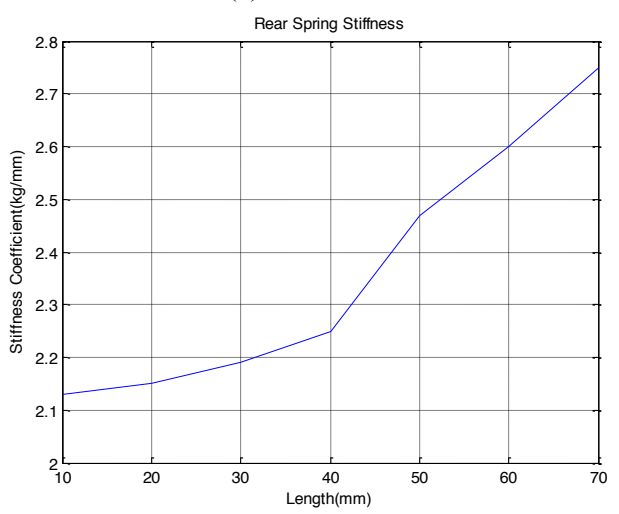

(b) Rear axle

Fig. 7. Spring stiffness.

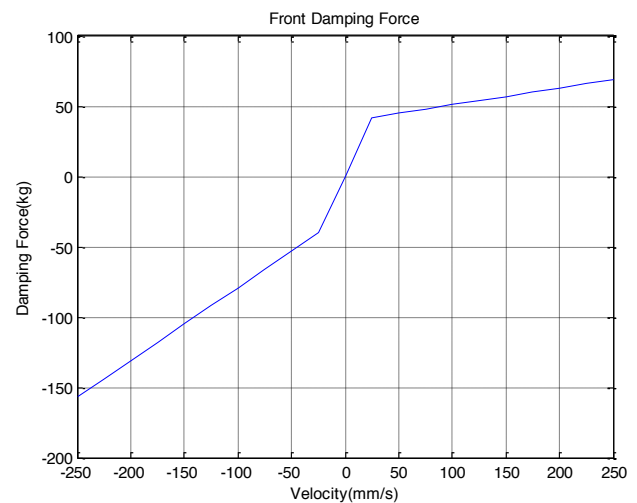

(a) Front axle

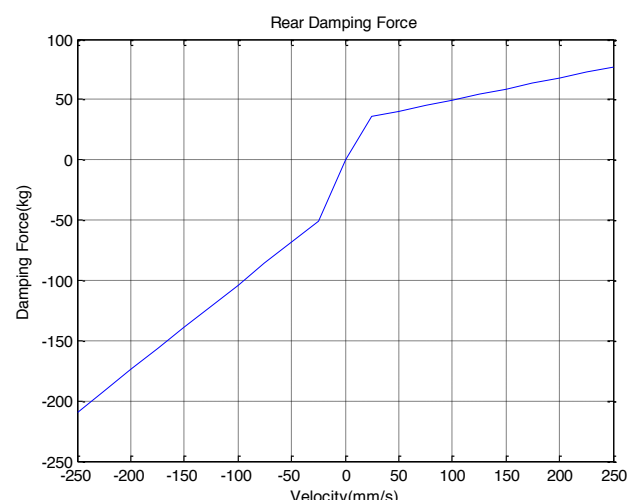

(b) Rear axle

Fig. 8. Damping characteristics of the shock absorbers. 


\subsection{Verification of the initial balanced positions of the suspension system}

To verify the initial balanced positions of the suspension system through the proposed mathematical model, the front axle suspension parameters (defined in Section 4.1) were substituted into (18) and (19) ; this enabled determining the initial lower control arm angle and spring deformation of the loaded suspension system in the balanced state. The identical parameter settings were applied to the ADAMS software tool to conduct simulation and obtain the simulated initial lower control arm angle and spring deformation.

In the mathematical model, the lower control arm angle shifted from $-7.964^{\circ}$ (the unloaded state) to $2.467^{\circ}$, turning counterclockwise by $10.431^{\circ}$. The shock absorber length decreased by $0.059 \mathrm{~m}$ from $0.5554 \mathrm{~m}$ to $0.4964 \mathrm{~m}$. Conversely, the ADAMS model determined that the lower control arm turned counterclockwise by $10.47^{\circ}$, and that the shock absorber length decreased by $0.05973 \mathrm{~m}$. Therefore, the two models attained similar results (Fig. 1), verifying the feasibility of the mathematical model proposed in this study.

Table 1. Comparison of the initial balanced positions determined with the proposed and ADAMS models.

\begin{tabular}{|c|c|c|}
\hline & $\begin{array}{c}\text { Change in the } \\
\text { lower control } \\
\text { arm angle }\end{array}$ & $\begin{array}{c}\text { Change in the } \\
\text { shock absorber } \\
\text { length }\end{array}$ \\
\hline ADAMS model & $10.470^{\circ}$ & $59.73 \mathrm{~mm}$ \\
\hline $\begin{array}{c}\text { Mathematical } \\
\text { model }\end{array}$ & $10.431^{\circ}$ & $59.0 \mathrm{~mm}$ \\
\hline
\end{tabular}

\subsection{Verification of the $1 / 4$ vehicle suspension mathematical models}

A chirp signal, which is a type of time-variant string signal available in MATLAB Simulink, with constant amplitude was adopted as the road input. In this study, the signal frequency was set as $0-20 \mathrm{~Hz}$ to conduct $40 \mathrm{~s}$ of simulation, as shown in Fig. 9. The vehicle body vertical acceleration, spring deformation, and tire deformation determined using the traditional, nonlinear, and linearized nonlinear models under specific road inputs were compared with those simulated using the ADAMS model.

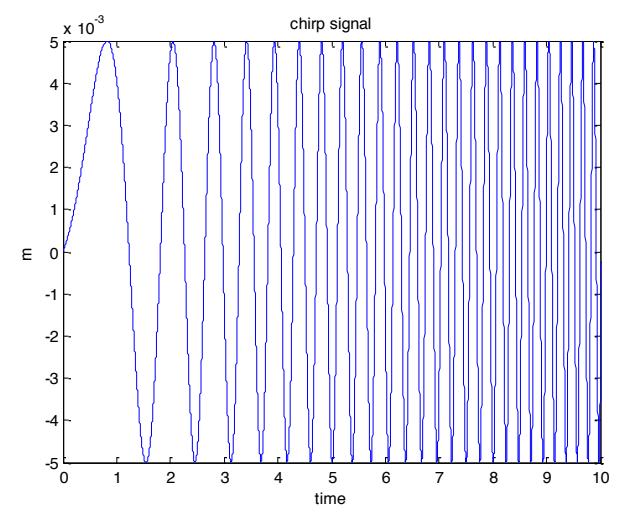

Fig. 9. Road input as a chirp signal.
Table 2. Mean values of the spring stiffness and shock absorber damping coefficient.

\begin{tabular}{|c|c|c|}
\hline & $\mathrm{k}(\mathrm{kg} / \mathrm{mm})$ & $\mathrm{c}(\mathrm{kg} \cdot \mathrm{s} / \mathrm{mm})$ \\
\hline Front axle & 2.6088 & 0.628 \\
\hline Rear axle & 2.8566 & 0.822 \\
\hline
\end{tabular}

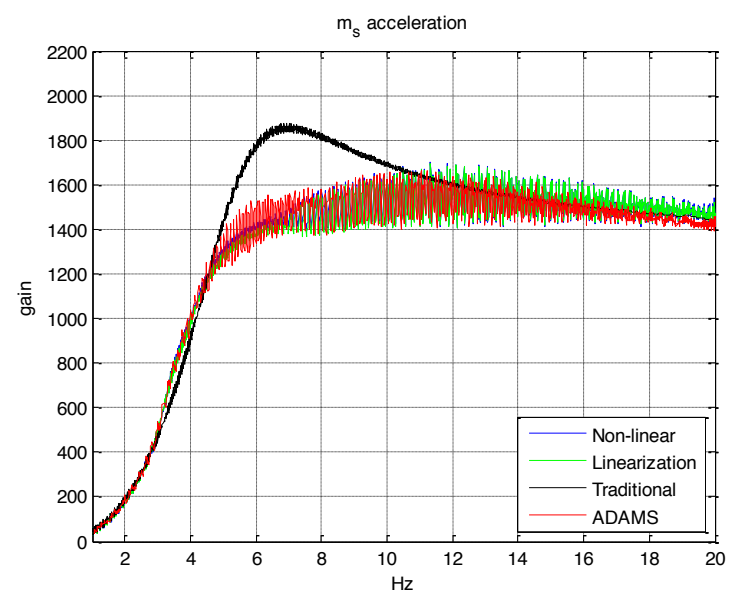

(a) Vehicle body vertical acceleration

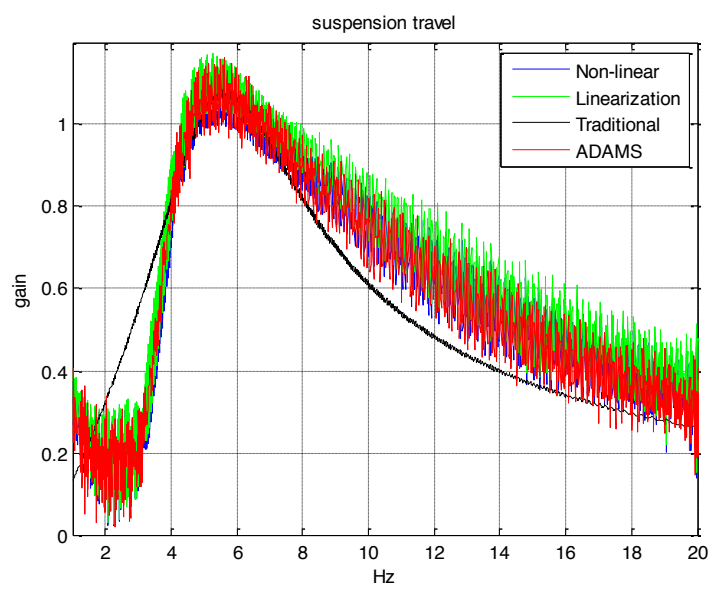

(b) Spring deformation

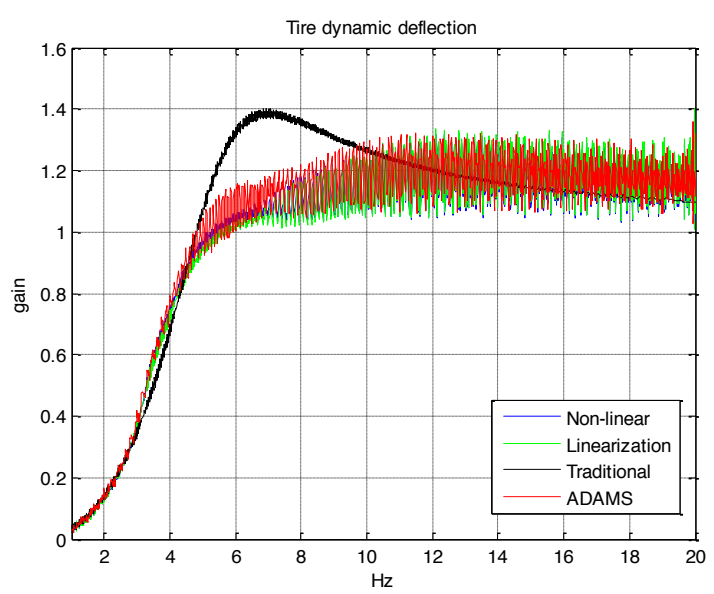

(c) Tire deformation

Fig. 10. Frequency responses resulting from the chirp signal road input. 
The front axle parameters were adopted. Specifically, the nonlinear, linearized nonlinear, and ADAMS model used the spring stiffness and shock absorber damping coefficients respectively displayed in Figs. 7(a) and 8(a). By contrast, the spring stiffness value of the traditional model yielded the mean tire rate obtained using the parameter simulation with the ADAMS suspension platform (Section 4.1), and the shock absorber damping coefficient was the mean value acquired from Fig. 8; Table 2 lists these parameter values. The nonlinear and linearized nonlinear models required determining the initial balanced positions before conducting simulation, whereas the time domain simulation involved the fast Fourier transform to obtain the frequency response (Fig. $10)$.

The simulation results (Fig. 10) showed that compared with the traditional model, the nonlinear and linearized nonlinear models demonstrated dynamic responses more similar to those of the ADAMS model, verifying that the proposed nonlinear mathematic model is more reliable than the traditional model. The vibration displayed in the simulation results resulted from the jump resonance of the nonlinear model, in which slight variations in the input frequency caused substantial fluctuation in the output amplitude. Although the nonlinear frequency responses are not applicable to comparing the response values under different frequencies, they still correspond with the results of the time domain analysis.

\subsection{Verification of the $1 / 2$ vehicle suspension mathematical models}

Fig. 11 presents the random road surface input built with the ADAMS software tool. Model verification was conducted using vehicle speeds of 10 and $15 \mathrm{~m} / \mathrm{s}$.

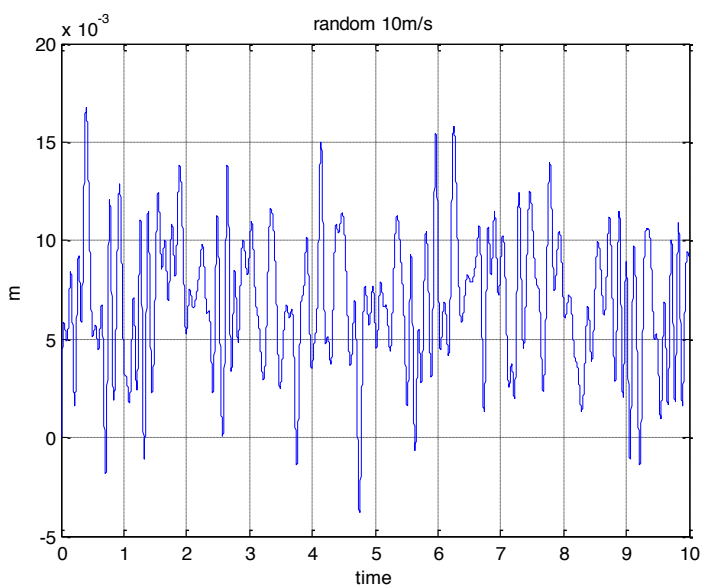

(a) Vehicle speed of $10 \mathrm{~m} / \mathrm{s}$

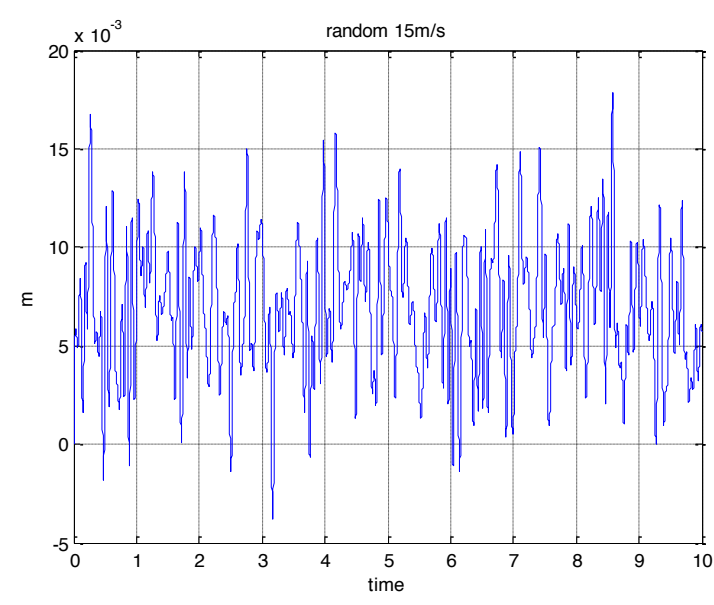

(b) Vehicle speed of $15 \mathrm{~m} / \mathrm{s}$

Fig. 11. Random road surface input.

The front and rear parameters of the nonlinear models were based on those defined in Section 4.1. The traditional model used the spring stiffness and shock absorber damping coefficient values displayed in Table 2 . Figs. 12 and 13 illustrate the simulation results.

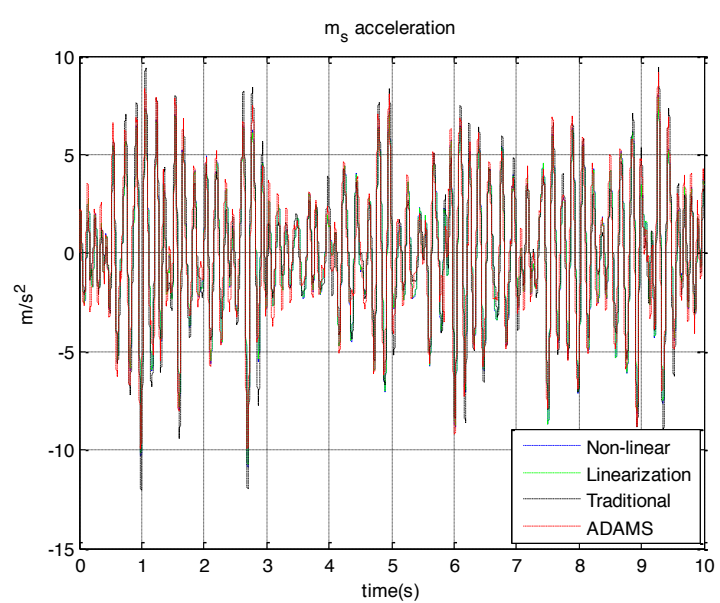

(a) Vertical acceleration at the vehicle center of mass

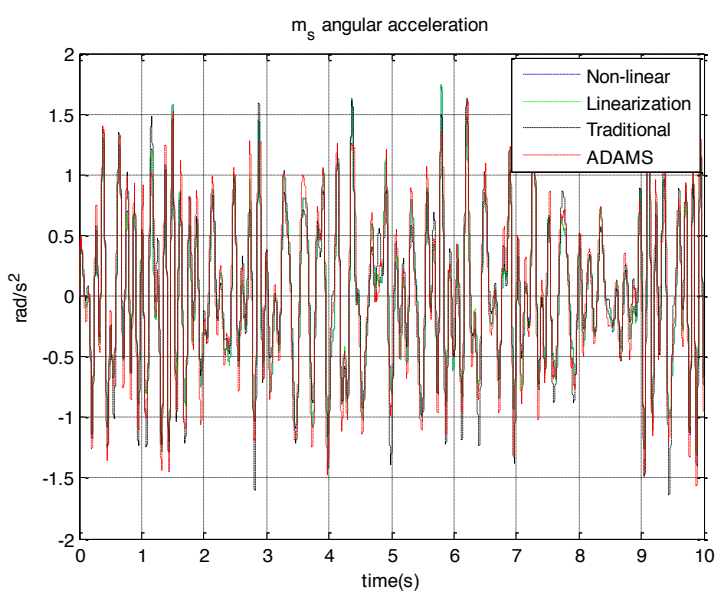

(b) Angular acceleration in the vehicle pitch direction

Fig. 12. Temporal responses at the vehicle speed of $10 \mathrm{~m} / \mathrm{s}$. 


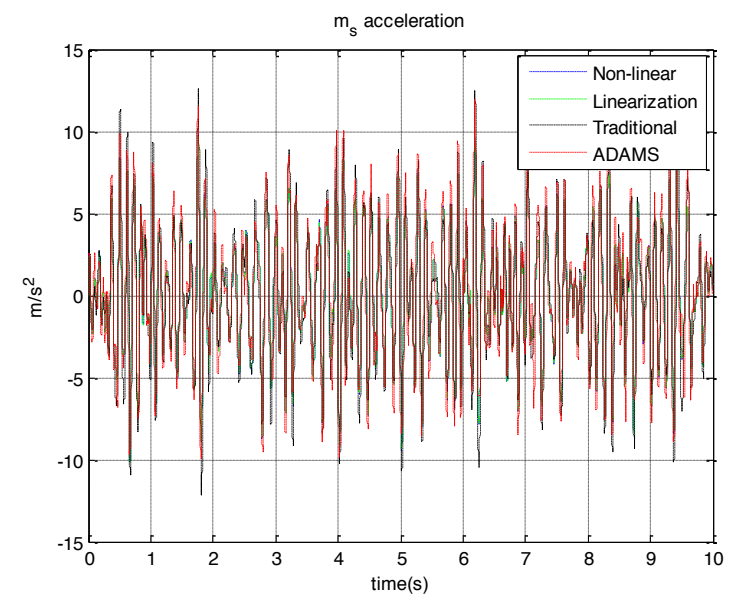

(a) Vertical acceleration at the vehicle center of mass

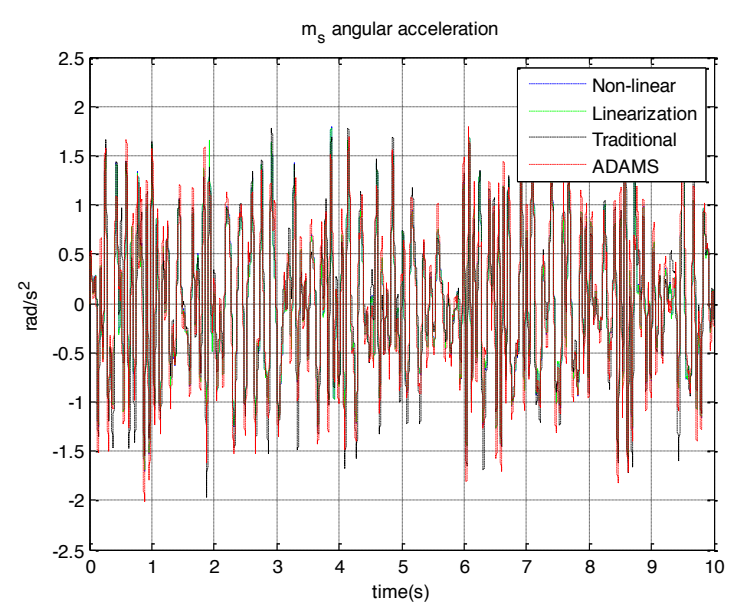

(b) Angular acceleration in the vehicle pitch direction

Fig. 13. Temporal responses at the vehicle speed of $15 \mathrm{~m} / \mathrm{s}$.

To evaluate the accuracy of the three models, the mean absolute differences (MADs) of the temporal responses obtained using the models were calculated and compared with those of the ADMAS model (Table 3). The MAD formula is expressed as (20).

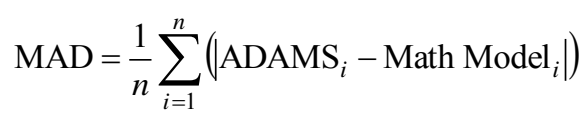

Table 3. Mean Absolute Difference.

\begin{tabular}{|c|c|c|c|c|}
\hline & & $\begin{array}{c}\text { Non- } \\
\text { Linear }\end{array}$ & $\begin{array}{c}\text { Lineari } \\
\text { zation }\end{array}$ & $\begin{array}{c}\text { Traditi } \\
\text { onal }\end{array}$ \\
\hline $\begin{array}{c}\text { Vertical } \\
\text { acceleration at the } \\
\text { vehicle center of } \\
\text { mass }\left(\mathrm{m} / \mathrm{s}^{2}\right)\end{array}$ & $10 \mathrm{~m} / \mathrm{s}$ & 0.8836 & 0.8822 & 1.0365 \\
\cline { 2 - 5 } & $15 \mathrm{~m} / \mathrm{s}$ & 0.9914 & 0.9864 & 1.2196 \\
\hline $\begin{array}{c}\text { Angular } \\
\text { acceleration in the } \\
\text { vehicle pitch } \\
\text { direction }\left(\mathrm{rad} / \mathrm{s}^{2}\right)\end{array}$ & $10 \mathrm{~m} / \mathrm{s}$ & 0.1284 & 0.1297 & 0.1572 \\
\cline { 2 - 5 } & $15 \mathrm{~m} / \mathrm{s}$ & 0.1598 & 0.1592 & 0.2191 \\
\hline
\end{tabular}

As shown in Table 3, the nonlinear and linearized nonlinear model attained results similar to those of the ADAMS model. By contrast, the results of the traditional model differed substantially from those of the ADAMS model, and the difference increased with vehicle speed. Comparing the $1 / 2$ models verified that the proposed nonlinear mathematical model is more accurate than the traditional model in reflecting the actual dynamic behavior of vehicles.

\section{Conclusions}

This study constructed a nonlinear model composed of suspension-related geometric parameters, and adopted static balance analysis and kinematics analysis to estimate the initial balanced positions of the suspension components subjected to various loads. This enabled comprehensively describing how the relative positions, velocity, and force transmission of the suspension components affect the motion of the vehicle body and tires. The nonlinear model was also linearized to develop control strategies for semi-active suspension systems. The analysis results showed that compared with the traditional model, the nonlinear and linearized nonlinear models attained simulation results more similar to those of the ADAMS model, verifying the accuracy of the proposed model. Furthermore, when the nonlinearity of the suspension system increased, the nonlinear and linearized nonlinear models effectively predicted the variations in the dynamic responses. Therefore, the proposed model can facilitate understanding the effect of suspension components on vehicle dynamic behavior to propose practicable control strategies.

\section{References}

1. R. Ramajani, Vehicle Dynamics and Control, Springer Science, New York, pp. 267-354, (2006)

2. J. Y. Wong, Theory of Ground Vehicles, John Wiley, pp. 461-520, (2008)

3. M. M. S. Kaldas, K. Çalışkan, R. Henze and F. Küçükay, "Development of a Semi-Active Suspension Controller Using Adaptive-Fuzzy with Kalman Filter" SAE Paper No. 2011-01-0431, (2011)

4. S. Rasal, J. .Jaganmohan, S. Agashe, K. P. Wani "Implementation of Fuzzy Logic Control in Semiactive Suspension for a Vehicle Using MATLAB SIMULINK" SAE Paper No. 2016-28-0035, (2016)

5. K. S. Hong, D. S. Jeon, W. S. Yoo, H. Sunwoo, S. Y. Shin, C. M. Kim, and B. S. Park, "A New Model and an Optimal Pole-Placement Control of the Macpherson Suspension System," SAE Technical Paper Series, 1999-01-1331, (1999)

6. W. F. Milliken and D. L. Milliken, Race Car Vehicle Dynamics, Society of Automotive Engineers, Inc., pp. 579-605, (1995)

7. M. Blundell and D. Harty, Multibody Systems Approach to Vehicle Dynamics, Elsevier Butterworth-Heinemann, pp. 185-334, (2015)

8. K. J. Waldron and G. L. Kinzel, Kinematics, Dynamics, and Design of Machinery, John Wiley \& Sons, pp. 319-354, (2016). 